\title{
Generalized Numerical Index and Denseness of Numerical Peak Holomorphic Functions on a Banach Space
}

\author{
Sung Guen Kim ${ }^{1}$ and Han Ju Lee \\ ${ }^{1}$ Department of Mathematics, Kyungpook National University, Daegu 702-701, Republic of Korea \\ ${ }^{2}$ Department of Mathematics Education, Dongguk University-Seoul, Seoul 100-715, Republic of Korea \\ Correspondence should be addressed to Han Ju Lee; hanjulee@dongguk.edu
}

Received 11 June 2013; Accepted 23 August 2013

Academic Editor: Geraldo Botelho

Copyright (c) 2013 S. G. Kim and H. J. Lee. This is an open access article distributed under the Creative Commons Attribution License, which permits unrestricted use, distribution, and reproduction in any medium, provided the original work is properly cited.

The generalized numerical index of a Banach space is introduced, and its properties on certain Banach spaces are studied. Ed-dari's theorem on the numerical index is extended to the generalized index and polynomial numerical index of a Banach space. The denseness of numerical strong peak holomorphic functions is also studied.

\section{Introduction and Preliminaries}

Let $X$ and $Y$ be Banach spaces over a scalar field $\mathbb{F}$, where $\mathbb{F}$ is the real field $\mathbb{R}$ or the complex field $\mathbb{C}$. We denote by $B_{X}$ and $S_{X}$ its closed unit ball and unit sphere, respectively. Let $X^{*}$ be the dual space of $X$. An $N$-homogeneous polynomial $P$ from $X$ to $Y$ is a mapping such that there is an $N$-linear (bounded) mapping $L$ from $X$ to $Y$ such that $P(x)=L(x, \ldots, x)$ for every $x$ in $X . \mathscr{P}\left({ }^{N} X: Y\right)$ denotes the Banach space of all $N$-homogeneous polynomials from $X$ to $Y$, endowed with the norm $\|P\|=\sup _{x \in B_{X}}\|P(x)\|$. A mapping $Q: X \rightarrow Y$ is a polynomial if there exist a nonnegative integer $m$ and $P_{k} \in \mathscr{P}\left({ }^{k} X: Y\right), k=0,1, \ldots, m$ such that $Q=P_{0}+P_{1}+\cdots+P_{m}$. If $P_{m} \neq 0$, then we say that $Q$ is a polynomial of degree $m$. We denote by $\mathscr{P}(X: Y)$ the normed space of all polynomials from $X$ to $Y$, endowed with the norm $\|Q\|=\sup _{x \in B_{X}}\|Q(x)\|$. We refer to [1] for background on polynomials on a Banach space.

For two Banach spaces $X, Y$ over a field $\mathbb{F}$ and a Hausdorff topological space $K$, let

$$
\begin{array}{r}
C_{b}(K: Y):=\{f: K \longrightarrow Y: f \text { be a bounded } \\
\text { continuous function on } K\} .
\end{array}
$$

Then $C_{b}(K: Y)$ is a Banach space under the sup norm $\|f\|:=$ $\sup \left\{\|f(t)\|_{Y}: t \in K\right\}$ and $\mathscr{P}\left({ }^{N} X: Y\right)$ is a closed subspace of
$C_{b}\left(B_{X}: Y\right)$ for each $N \geq 1$. We just write $C_{b}(K)$ and $\mathscr{P}\left({ }^{N} X\right)$ instead of $C_{b}(K: \mathbb{F})$ and $\mathscr{P}\left({ }^{N} X: \mathbb{F}\right)$, respectively.

For complex Banach spaces $X$ and $Y$, we denote that

$$
\begin{gathered}
A_{b}\left(B_{X}: Y\right):=\left\{f \in C_{b}\left(B_{X}: Y\right): f \text { is holomorphic on } B_{X}^{\circ}\right\} \\
A_{u}\left(B_{X}: Y\right):=\left\{f \in A_{b}\left(B_{X}: Y\right): f\right. \text { is } \\
\text { uniformly continuous }\}
\end{gathered}
$$

where $B_{X}^{\circ}$ is the interior of $B_{X}$. Then $A_{b}\left(B_{X}: Y\right)$ and $A_{u}\left(B_{X}\right.$ : $Y)$ are closed subspaces of $C_{b}\left(B_{X}: Y\right)$. In case that $Y$ is the complex scalar field $\mathbb{C}$, we write $A_{b}\left(B_{X}\right)$ and $A_{u}\left(B_{X}\right)$ instead of $A_{b}\left(B_{X}: Y\right)$ and $A_{u}\left(B_{X}: Y\right)$, respectively. The closed subspace of $A_{u}\left(B_{X}: Y\right)$ consisting of all weakly uniformly continuous functions is denoted by $A_{w u}\left(B_{X}: Y\right)$. We denote by $A\left(B_{X}: X\right)$ one of $A_{b}\left(B_{X}: X\right), A_{u}\left(B_{X}: X\right)$, and $A_{w u}\left(B_{X}:\right.$ $X)$. Notice that if $X$ is finite dimensional, $A_{b}\left(B_{X}: X\right)=$ $A_{u}\left(B_{X}: X\right)=A_{w u}\left(B_{X}: X\right)$.

Given a real or complex Banach space $X$, we denote by $\tau$ the product topology of the set $S_{X} \times S_{X^{*}}$, where the topologies on $S_{X}$ and $S_{X^{*}}$ are the norm topology of $X$ and the weak-* topology of $X^{*}$, respectively. The set $\Pi(X):=\left\{\left(x, x^{*}\right) \in S_{X} \times\right.$ $\left.S_{X^{*}}:\|x\|=\left\|x^{*}\right\|=1=x^{*}(x)\right\}$ is a $\tau$-closed subset of $S_{X} \times S_{X^{*}}$. The spatial numerical range of $f$ in $C_{b}\left(B_{X}: X\right)$ is defined [2] by $W(f)=\left\{x^{*}(f(x)):\left(x, x^{*}\right) \in \Pi(X)\right\}$, and the numerical 
radius of $f$ is defined by $v(f)=\sup \{|\lambda|: \lambda \in W(f)\}$. Let $f$ be an element of $C_{b}(K: X)$. We say that fattains its norm if there is some $t \in K$ such that $\|f\|=\|f(t)\|_{X} . f$ is said to be a (norm) peak function at $t$ if there exists a unique $t \in K$ such that $\|f\|=\|f(t)\|_{X}$. It is clear that every (norm) peak function in $C_{b}(K: X)$ is norm attaining. A peak function $f$ at $t$ is said to be a (norm) strong peak function if whenever there is a sequence $\left\{t_{k}\right\}_{k=1}^{\infty}$ in $K$ with $\lim _{k}\left\|f\left(t_{k}\right)\right\|_{X}=\|f\|$, $\left\{t_{k}\right\}_{k=1}^{\infty}$ converges to $t$ in $K$. It is easy to see that if $K$ is compact, then every peak function is a strong peak function. Given a subspace $H$ of $C_{b}(K)$, we denote by $\rho H$ the set of all points $t \in K$ such that there is a strong peak function $f$ in $H$ with $\|f\|=|f(t)|$.

Similarly we introduce the notion of numerical peak functions. Let $f$ be an element of $C_{b}\left(B_{X}: X\right)$. If there is some $\left(x, x^{*}\right) \in \Pi(X)$ such that $v(f)=\left|x^{*}(f(x))\right|$, we say [3] that $f$ attains its numerical radius. $f$ is said $([4,5])$ to be a numerical peak function at $\left(x, x^{*}\right)$ if there exist a unique $\left(x, x^{*}\right) \in \Pi(X)$ such that $v(f)=\left|x^{*}(f(x))\right|$. In this case, $\left(x, x^{*}\right)$ is said to be the numerical peak point of $f$. It is clear that every numerical peak function in $C_{b}\left(B_{X}: X\right)$ is numerical radius attaining. The numerical peak function $f$ at $\left(x, x^{*}\right)$ is called a numerical strong peak function if whenever there is a sequence $\left\{\left(x_{k}, x_{k}^{*}\right)\right\}_{k=1}^{\infty}$ in $\Pi(X)$ such that $\lim _{k}\left|x_{k}^{*}\left(f\left(x_{k}\right)\right)\right|=v(f)$, then $\left\{\left(x_{k}, x_{k}^{*}\right)\right\}_{k=1}^{\infty}$ converges to $\left(x, x^{*}\right)$ in $\tau$-topology. In this case, $\left(x, x^{*}\right)$ is said to be the numerical strong peak point of $f$. We say that a numerical strong peak function $f$ at $\left(x, x^{*}\right)$ is said to be a very strong numerical peak function if whenever there is a sequence $\left\{\left(x_{k}, x_{k}^{*}\right)\right\}_{k=1}^{\infty}$ in $\Pi(X)$ satisfying $\lim _{n}\left|x_{k}^{*}\left(f\left(x_{k}\right)\right)\right|=v(f)$, we get $\lim _{k} x_{k}=x$ and $\lim _{k} x_{k}^{*}=x^{*}$ in the norm topology. If $X$ is finite dimensional, then every numerical peak function is a very strong numerical peak function.

In 1996, Choi and Kim [6] initiated the study of denseness of norm or numerical radius attaining nonlinear functions, especially homogeneous polynomials on a Banach space. Using the perturbed optimization theorem of Bourgain [7] and Stegall [8], they proved that if a real or complex Banach space $X$ has the Radon-Nikodým property, then the set of all norm attaining functions in $\mathscr{P}\left({ }^{k} X\right)$ is norm-dense. For the definition and properties of the Radon-Nikodým property, see [9]. Concerning the numerical radius, it was also shown that if $X$ has the Radon-Nikodým property, then the set of all numerical radii attaining functions in $\mathscr{P}\left({ }^{k} X: X\right)$ is normdense. Acosta et al. [10] proved that if a complex Banach space $X$ has the Radon-Nikodým property, then the set of all norm attaining functions in $A_{b}\left(B_{X}\right)$ is norm-dense. Recently, it was shown in [11] that if $X$ has the Radon-Nikodým property, the set of all (norm) strong peak functions in $A_{b}\left(B_{X}\right)$ is dense. Concerning the numerical radius, Acosta and Kim [3] showed that the set of all numerical radii attaining functions in $A_{b}\left(B_{X}: X\right)$ is dense if $X$ has the Radon-Nikodým property. When $X$ is a smooth (complex) Banach space with the Radon-Nikodým property, it is shown in [5] that the set of all numerical strong peak functions is dense in $A\left(B_{X}: X\right)$. As a corollary, if $1<p<\infty$ and $X=L_{p}(\mu)$ for a measure space $\mu$, then the set of all norm and numerical strong peak functions in $A\left(B_{X}: X\right)$ is a dense $G_{\delta}$-subset of $A\left(B_{X}: X\right)$.
In this case, every numerical strong peak function is a very strong numerical peak function. It is also shown in [5] that the set of all norm and numerical strong peak functions in $A\left(B_{l_{1}}: l_{1}\right)$ is a dense $G_{\delta}$-subset of $A\left(B_{l_{1}}: l_{1}\right)$.

Let us briefly sketch the content of this paper. In Section 2 , to extend the results of a finite dimensional space to an infinite dimensional space by approximation, we introduce the following notions. A Banach space $X$ has the (FPA)property with $\left\{\pi_{i}, F_{i}\right\}_{i \in I}$ if

(1) each $\pi_{i}$ is a norm-one projection with the finite dimensional range $F_{i}$,

(2) given $\epsilon>0$, for every finite-rank operator $T$ from $X$ into a Banach space $F$ and for every finite dimensional subspace $G$ of $X$, there is $\pi_{i}$ such that

$$
\left\|T-T \pi_{i}\right\| \leq \epsilon, \quad\left\|I_{G}-\left.\pi_{i}\right|_{G}\right\| \leq \epsilon .
$$

As examples, we show that $X$ has the (FPA)-property if at least one of the following conditions is satisfied.

(a) It has a shrinking and monotone finite-dimensional decomposition.

(b) $X=L_{p}(\mu)$, where $\mu$ is a finite measure and $1 \leq p<$ $\infty$.

We show that if $X$ has the (FPA)-property, then the set of all polynomials $Q \in \mathscr{P}(X: X)$ such that there exist a finite dimensional subspace $F$ and norm-one projection $\pi: X \rightarrow F$ such that $\pi \circ Q \circ \pi=Q$ and $\left.Q\right|_{F}$ is a norm, and numerical peak function as a mapping from $B_{F}$ into $F$ is dense in $A_{w u}\left(B_{X}: X\right)$.

A subset $\Gamma$ of $\Pi(X)$ is called a numerical boundary for a subspace $H$ of $C_{b}\left(B_{X}: X\right)$ if $v(f)=\sup \left\{\left|x^{*}(f(x))\right|\right.$ : $\left.\left(x, x^{*}\right) \in \Gamma\right\}$ for every $f \in H$ (see $\left.[4,12]\right)$. The projections $\left\{\pi_{i}, F_{i}\right\}_{i \in I}$ are said to be parallel to a numerical boundary $\Gamma$ of $H$ if each $\pi_{i}$ has the image $F_{i}$ and

$$
\left|\left\langle\left. x^{*}\right|_{F_{i}}, \pi_{i}(x)\right\rangle\right|=\left\|\left.x^{*}\right|_{F_{i}}\right\| \cdot\left\|\pi_{i}(x)\right\|, \quad \forall\left(x, x^{*}\right) \in \Gamma, \forall i \in I .
$$

A projection $\pi: X \rightarrow X$ is said to be strong if whenever $\left\{\pi\left(x_{k}\right)\right\}_{k=1}^{\infty}$ is norm-convergent to $y \in S_{X}$ for a sequence $\left\{x_{k}\right\}_{k=1}^{\infty}$ in $B_{X},\left\{x_{k}\right\}_{k=1}^{\infty}$ is norm-convergent to $y$.

Recall that a Banach space $X$ is said to be locally uniformly convex if $x \in S_{X}$, and there is a sequence $\left\{x_{n}\right\}$ in $B_{X}$ satisfying $\lim _{n}\left\|x_{n}+x\right\|=2$, then $\lim _{n}\left\|x_{n}-x\right\|=0$. Notice that if $X$ is locally uniformly convex, then every norm-one projection is strong. We prove that if a smooth Banach space $X$ has the (FPA)-property and the corresponding projections are strong and parallel to $\Pi(X)$, then the set of all norm and numerical strong peak functions in $A_{w u}\left(B_{X}: X\right)$ is dense. We also prove that if a Banach space $X$ has the (FPA)-property with $\left\{\left(\pi_{i}, F_{i}\right)\right\}_{i \in I}$, the corresponding projections are strong, parallel to $\Pi(X)$, and if each $\pi_{i}^{*}: X^{*} \rightarrow X^{*}$ is strong, then the set of all very strong numerical and norm strong peak functions is dense in $A_{w u}\left(B_{X}: X\right)$.

In Section 3, we extend the recent result of Ed-dari [13]. Let $X$ be a complex Banach space and $H$ a subspace of 
$A_{b}\left(B_{X}: X\right)$. We introduce the $H$-numerical index by $N(H):=\inf \{v(f): f \in H,\|f\|=1\}$. When $H=\mathscr{P}\left({ }^{k} X: X\right)$ for some $k \geq 1$, the polynomial numerical index $N(H)$ is usually denoted by $n^{(k)}(X)$, which was first introduced and studied by Choi et al. [14]. We refer to [15-20] for some recent results about polynomial numerical index. For a normone projection $\pi$ with range $F$ and for any subspace $H$ of $A_{b}\left(B_{X}: X\right)$, define $H_{F}=\left\{\left.\pi \circ f \circ \pi\right|_{F}: B_{F} \rightarrow F: f \in H\right\}$. We prove that if $X$ has the (FPA)-property with $\left\{\left(\pi_{i}, F_{i}\right)\right\}_{i \in I}$ and the corresponding projections are parallel to a numerical boundary of a subspace $H$, then $N(H)=\inf _{i \in I} N\left(H_{F_{i}}\right)$. In fact, $N(H)$ is a decreasing limit of the right-hand side with respect to the inclusion partial order. If $X$ is a real Banach space, we get a similar result (see Theorem 14). As a corollary we also extended Ed-dari's result to the polynomial numerical indices of $l_{p}$. In fact, Kim [17] extended Ed-dari's result [13, Theorem 2.1] to the polynomial numerical indices of (real or complex) $l_{p}$ of order $k$ as follows: Let $1<p<\infty$ and $k \in \mathbb{N}$ be fixed. Then $n^{(k)}\left(l_{p}\right)=\inf \left\{n^{(k)}\left(l_{p}^{m}\right): m \in \mathbb{N}\right\}$ and the sequence $\left\{n^{(k)}\left(l_{p}^{m}\right)\right\}_{m \in \mathbb{N}}$ is decreasing.

\section{Banach Spaces with the (FPA)-Property and Denseness of Numerical Peak Holomorphic Functions}

Following [21, Definition 1.g.1], a Banach space $X$ has a finitedimensional Schauder decomposition (FDD for short) if there is a sequence $\left\{X_{n}\right\}$ of finite-dimensional spaces such that every $x \in X$ has a unique representation of the form $x=$ $\sum_{n=1}^{\infty} x_{n}$, where $x_{n} \in X_{n}$ for every $n$. In such a case, the projections given by $P_{n}(x)=\sum_{i=1}^{n} x_{i}$ are linear and bounded operators. If, moreover, for every $x^{*} \in X^{*}$, it is satisfied that $\left\|P_{n}^{*} x^{*}-x^{*}\right\| \rightarrow 0$, the FDD is called shrinking. The FDD is said to be monotone if $\left\|P_{n}\right\|=1$ for every $n$.

The following proposition is easy to prove and its proof is omitted.

Proposition 1. The following two conditions on a Banach space are equivalent.

(1) A Banach space X has the (FPA)-property.

(2) Given $\epsilon>0,\left\{x_{1}, \ldots, x_{m}\right\} \subset X$ and $\left\{x_{1}^{*}, \ldots, x_{n}^{*}\right\} \subset X^{*}$, there is a norm-one projection $P: X \rightarrow X$ such that $P$ has a finite rank, and for each $i=1, \ldots, m$ and for each $j=1, \ldots, n$, there exist $y_{i} \in X$ and $y_{j}^{*} \in X^{*}$ such that $\left\|P y_{i}-x_{i}\right\| \leq \epsilon$ and $\left\|P^{*} y_{j}^{*}-x_{j}^{*}\right\| \leq \epsilon$.

Example 2. Assume that $X$ is a complex Banach space satisfying at least one of the following conditions.

(1) It has a shrinking and monotone finite-dimensional decomposition.

(2) $X=L_{p}(\mu)$, where $\mu$ is a finite measure and $1 \leq p<$ $\infty$.

Then $X$ has the (FPA)-property.

Proof. Let $T: X \rightarrow F$ be a linear operator from $X$ to a finite dimensional space $F$ and $G$ a finite dimensional subspace $G$ of $X$. Given $\epsilon>0$, there is an $\epsilon / 3$-net $\left\{g_{1}, \ldots, g_{n}\right\}$ in $B_{G}$ and $T$ can be written as $\sum_{i=1}^{m} x_{i}^{*} \otimes y_{i}$ for some $x_{1}^{*}, \ldots, x_{m}^{*} \in X^{*}$ and $y_{1}, \ldots, y_{m} \in F$.

(1) Suppose that $X$ has a shrinking monotone finitedimensional decomposition. Then there is $N \in \mathbb{N}$ such that

$$
\max _{1 \leq i \leq m}\left\|y_{i}\right\| \cdot \sum_{i=1}^{m}\left\|P_{N}^{*} x_{i}^{*}-x_{i}^{*}\right\| \leq \epsilon, \quad \max _{1 \leq j \leq n}\left\|P_{N} g_{j}-g_{j}\right\| \leq \frac{\epsilon}{3} \text {. }
$$

Then for any $x \in B_{X}$,

$$
\begin{aligned}
\left\|T P_{N} x-T x\right\| & =\left\|\sum_{i=1}^{m}\left(P_{N}^{*} x_{i}^{*}\right)(x) y_{i}-\sum_{i=1}^{m} x_{i}^{*}(x) y_{i}\right\| \\
& \leq \max _{1 \leq j \leq n}\left\|y_{i}\right\| \cdot \sum_{i=1}^{m}\left\|P_{N}^{*} x_{i}^{*}-x_{i}^{*}\right\| \leq \epsilon,
\end{aligned}
$$

hence $\left\|T P_{N}-T\right\| \leq \epsilon$. For any $x \in B_{G}$, there is $g_{j}$ such that $\left\|x-g_{j}\right\| \leq \epsilon / 3$, then because the decomposition is monotone,

$$
\begin{aligned}
\left\|P_{N} x-x\right\| & \leq\left\|P_{N}\left(x-g_{j}\right)\right\|+\left\|P_{N} g_{j}-g_{j}\right\|+\left\|x-g_{j}\right\| \\
& \leq 2\left\|x-g_{j}\right\|+\left\|P_{N} g_{j}-g_{j}\right\| \leq \epsilon .
\end{aligned}
$$

So taking $P=P_{N}$, we obtained the desired result.

(2) Suppose that $X=L^{p}(\mu)$. We may assume that $\mu$ is a probability measure. For each $1 \leq i \leq m$, there is $s_{i} \in L_{q}(\mu)$ such that $1 / p+1 / q=1$ and $x_{i}^{*}(f)=\int f s_{i} d \mu\left(f \in L_{p}(\mu)\right)$. Then there is a sub- $\sigma$-algebra $\mathscr{F}$ generated by finite disjoint subsets such that

$$
\begin{gathered}
\max _{1 \leq j \leq n}\left\|y_{i}\right\| \cdot \sum_{i=1}^{m}\left\|E\left(s_{i} \mid \mathscr{F}\right)-s_{i}\right\|_{q} \leq \frac{\epsilon}{2}, \\
\max _{1 \leq i \leq n}\left\|E\left(g_{i} \mid \mathscr{F}\right)-g_{i}\right\|_{p} \leq \frac{\epsilon}{3} .
\end{gathered}
$$

Define a projection $P: X \rightarrow X$ as $P f=E(f \mid \mathscr{F})$. It is clear that $P$ is a norm-one projection. For any $f \in B_{X}$,

$$
\begin{aligned}
\|T P f-T f\|= & \left\|\sum_{i=1}^{m}\left(x_{i}^{*}\right)(P f) y_{i}-\sum_{i=1}^{m} x_{i}^{*}(f) y_{i}\right\| \\
\leq & \max _{1 \leq j \leq n}\left\|y_{i}\right\| \cdot \sum_{i=1}^{m}\left|x_{i}^{*}(P f)-x_{i}^{*}(f)\right| \\
\leq & \max _{1 \leq j \leq n}\left\|y_{i}\right\| \\
& \cdot \sum_{i=1}^{m}\left|\int_{K}(E(f \mid \mathscr{F})-f) E\left(s_{i} \mid \mathscr{F}\right) d \mu\right| \\
& +\max _{1 \leq j \leq n}\left\|y_{i}\right\| \\
& \cdot \sum_{i=1}^{m}\left|\int_{K}(E(f \mid \mathscr{F})-f)\left(E\left(s_{i} \mid \mathscr{F}\right)-s_{i}\right) d \mu\right|
\end{aligned}
$$




$$
\begin{aligned}
& =0+\max _{1 \leq j \leq n}\left\|y_{i}\right\| \\
& \cdot \sum_{i=1}^{m} \mid \int_{K}(E(f \mid \mathscr{F})-f) \\
& \quad \times\left(E\left(s_{i} \mid \mathscr{F}\right)-s_{i}\right) d \mu \mid \\
& \leq \max _{1 \leq j \leq n}\left\|y_{i}\right\| \cdot 2 \sum_{i=1}^{m}\|f\|_{p}\left\|E\left(s_{i} \mid \mathscr{F}\right)-s_{i}\right\|_{q} \leq \epsilon .
\end{aligned}
$$

On the other hand, for any $f \in B_{G}$, there is $g_{j}$ such that $\| f-$ $g_{j} \| \leq \epsilon / 3$. So

$$
\begin{aligned}
\|P f-f\| & \leq\left\|P\left(f-g_{j}\right)\right\|+\left\|P g_{j}-g_{j}\right\|+\left\|x-g_{j}\right\| \\
& \leq 2\left\|f-g_{j}\right\|+\left\|P g_{j}-g_{j}\right\| \leq \epsilon .
\end{aligned}
$$

We obtained the desired result. The proof is complete.

We will say that a $k$-linear mapping $L: X \times \cdots \times X \rightarrow Y$ is of finite-type if it can be written as

$$
L\left(x_{1}, \ldots, x_{k}\right)=\sum_{i=1}^{m} x_{1, i}^{*}\left(x_{1}\right) \cdots x_{k, i}^{*}\left(x_{k}\right) y_{i}, \quad \forall x_{1}, \ldots, x_{k} \in X
$$

for some $m \in \mathbb{N}, x_{1,1}^{*}, \ldots, x_{k, m}^{*}$ in $X^{*}$ and $y_{1}, \ldots, y_{m}$ in $Y$. We will denote by $L_{f}\left({ }^{k} X: Y\right)$ the space of all $k$-linear mappings from $X$ to $Y$ of finite type. If a polynomial $P$ is associated with such a $k$-linear mapping, we will say that it is a finitetype polynomial.

Proposition 3. Suppose that a Banach space X has the (FPA)property with $\left\{\left(\pi_{i}, F_{i}\right)\right\}_{i}$. Then the set of all polynomials $Q \in$ $\mathscr{P}(X: X)$ such that there exists a projection $\pi_{i}: X \rightarrow F_{i}$ such that $\pi_{i} \circ Q \circ \pi_{i}=Q$ and $\left.Q\right|_{F_{i}}$ is a norm and numerical peak function as a mapping from $B_{F_{i}}$ to $F_{i}$ is dense in $A_{w u}\left(B_{X}: X\right)$.

Proof. We follow the ideas in [10]. The subset of continuous polynomials is always dense in $A_{u}\left(B_{X}: X\right)$. Given $f \in$ $A_{u}\left(B_{X}: X\right)$ and $n \in \mathbb{N}$, it is the limit in $A_{u}\left(B_{X}: X\right)$ of sequence of functions $\left\{f_{n}\right\}_{n}$ defined by $f_{n}(x):=f((n /(n+$ $1)) x$ ). Then $f_{n}$ belongs to $A_{b}\left(((n+1) / n) B_{X}: X\right)$. Thus the Taylor series expansion of $f_{n}$ at 0 converges uniformly on $B_{X}$ for all $n$.

We will also use the fact that if $\sum_{k=0}^{\infty} P_{k}$ is the Taylor series expansion of $f \in A_{w u}\left(B_{X}: X\right)$ at 0 , then $P_{k}$ is weakly uniformly continuous on $B_{X}$ for all $k$.

Since $X$ has the (FPA)-property, $X^{*}$ has the approximation property (see [22, Lemma 3.1]). Then the subspace of $k$-homogeneous polynomials of finite-type restricted on $B_{X}$ is dense in the subspace of all $k$-homogeneous polynomials which are weakly uniformly continuous on $B_{X}$ (see $[1$, Proposition 2.8]). Thus the subspace of the polynomials of finite-type restricted to the closed unit ball of $X$ is dense in $A_{w u}\left(B_{X}: X\right)$.

Assume that $P$ is a finite-type polynomial that can be written as a finite sum $P=\sum_{k=0}^{n} P_{k}$, where each $P_{k}$ is an homogeneous finite-type polynomial with degree $k$. Consider the symmetric $k$-linear form $A_{k}$ associated with the corresponding polynomial $P_{k}$. Since $P_{k}$ is a finite-type polynomial, then $T_{k}: X \rightarrow L_{f}\left({ }^{k-1} X: X\right)$ given by

$$
T_{k}(x)\left(x_{1}, \ldots, x_{k-1}\right):=A_{k}\left(x, x_{1}, \ldots, x_{k-1}\right), \quad \forall x \in X
$$

is a linear finite-rank operator for any $1 \leq k \leq n$.

The direct sum of these operators, that is, the operator

$$
T: X \longrightarrow \bigoplus_{k=1}^{n} L_{f}\left({ }^{k-1} X: X\right)
$$

given by $T(x):=\left(T_{1}(x), \ldots, T_{n}(x)\right)$, for all $x \in X$, is also of finite rank.

By the assumption on $X$, given any $\epsilon>0$, there is a normone projection $\pi:=\pi_{i}: X \rightarrow X$ with a finite-dimensional range such that $\|T-T \pi\| \leq \epsilon$ and $\left\|\left.\pi\right|_{G}-I_{G}\right\| \leq \epsilon$, where $G$ is the span of $\bigcup_{k=1}^{n} P_{k}(X)$.

Let $B_{k}$ be the symmetric $k$-linear mapping given by $B_{k}:=$ $A_{k} \circ(\pi, \ldots, \pi)$, and let $Q_{k}$ be the associated polynomial. It happens that $Q_{k}=P_{k} \circ \pi$. Now for $\|x\| \leq 1$, we have

$$
\begin{aligned}
& \left\|P_{k} \circ \pi(x)-P_{k}(x)\right\| \\
& =\left\|\sum_{j=0}^{k-1}\left(\begin{array}{l}
k \\
j
\end{array}\right) A_{k}\left((x-\pi(x))^{k-j}, \pi(x)^{j}\right)\right\| \\
& =\left\|\sum_{j=0}^{k-1}\left(\begin{array}{l}
k \\
j
\end{array}\right)\left(T_{k}-T_{k} \circ \pi\right)(x)\left((x-\pi(x))^{k-j-1}, \pi(x)^{j}\right)\right\| \\
& \leq \sum_{j=0}^{k-1}\left(\begin{array}{l}
k \\
j
\end{array}\right)\left\|T_{k}-T_{k} \circ \pi\right\|\|x\|\|x-\pi(x)\|^{k-j-1}\|\pi(x)\|^{j} \\
& \leq \epsilon \sum_{j=0}^{k-1}\left(\begin{array}{c}
k \\
j
\end{array}\right) 2^{k-j-1} \leq 4^{k} \epsilon .
\end{aligned}
$$

Then $\left\|P_{k} \circ \pi-P_{k}\right\| \leq 4^{k} \epsilon$ and

$$
\begin{aligned}
& \left\|\pi \circ P_{k} \circ \pi-P_{k}\right\| \\
& \quad \leq\left\|\pi \circ P_{k} \circ \pi-\pi \circ P_{k}\right\|+\left\|\pi \circ P_{k}-P_{k}\right\| \leq 2 \cdot 4^{k} \epsilon .
\end{aligned}
$$

Let $R_{k}=\pi \circ P_{k} \circ \pi$ and $R=P_{0}+\sum_{k=1}^{n} R_{k}$. Then $\|R-P\| \leq$ $2 n 4^{n} \epsilon$. By [5, Theorem 2.9], there is a numerical and norm peak polynomial $Q^{\prime}: \pi(X) \rightarrow \pi(X)$ of degree $\leq n$ such that $\left\|\left.R\right|_{\pi(X)}-Q^{\prime}\right\| \leq \epsilon$. Setting $Q:=Q^{\prime} \circ \pi,\|P-Q\| \leq\left(2 n 4^{n}+2\right) \epsilon$. The proof is done.

Remark 4. If $X$ is a Banach space satisfying the (FPA)property, then the set of polynomials in $B_{A_{w u}\left(B_{X}: X\right)}$ which has a nontrivial invariant subspace and has a fixed point is dense in $B_{A_{w u}\left(B_{X}: X\right)}$.

Notice that if $X$ is locally uniformly convex, then every norm-one projection is strong. Indeed, suppose that if $\pi$ : 
$X \rightarrow F$ is a norm-one projection and if $\left\{\pi\left(x_{k}\right)\right\}_{k=1}^{\infty}$ in $B_{X}$ converges to $y \in S_{F}$, then

$$
\begin{aligned}
1 & =\lim _{k}\left\|\frac{\pi\left(x_{k}\right)+y}{2}\right\| \\
& =\lim _{k}\left\|\frac{\pi\left(x_{k}+y\right)}{2}\right\| \leq \lim _{k}\left\|\frac{x_{k}+y}{2}\right\| \leq 1
\end{aligned}
$$

shows that $\lim _{k}\left\|x_{k}+y\right\|=2$ and $\lim _{k}\left\|x_{k}-y\right\|=0$ since $X$ is locally uniformly convex.

The following lemma is proved in [5].

Lemma 5 (see [5]). Let $X$ be a complex Banach space and $f \in$ $A_{b}\left(B_{X}: X\right)$. Suppose that there are $y \in B_{X}$ and $y^{*} \in B_{X^{*}}$ such that $\left|y^{*}(y)\right|=\left\|y^{*}\right\| \cdot\|y\|$. Then $\left|y^{*}(f(y))\right| \leq v(f)$. In particular, $\|f(0)\| \leq v(f)$.

Theorem 6. Suppose that a smooth Banach space $X$ has the (FPA)-property with $\left\{\pi_{i}, F_{i}\right\}_{i \in I}$ and the corresponding projections are strong and parallel to $\Pi(X)$. Then the set of all numerical and norm strong peak functions in $A_{w u}\left(B_{X}: X\right)$ is dense.

Proof. By Proposition 3, the set of all polynomials $Q$ such that there exists norm-one projection $\pi:=\pi_{i}: X \rightarrow F$ such that $\pi \circ Q \circ \pi=Q$ and $\left.Q\right|_{F}$ is a norm and numerical peak function as a mapping from $B_{F}$ to $F$ is dense in $A_{w u}\left(B_{X}: X\right)$.

Fix corresponding $Q$ and $\pi$ and assume that $v_{F}(Q)=$ $\left|y_{0}^{*}\left(Q\left(y_{0}\right)\right)\right|$ and $\left\|Q\left(y_{1}\right)\right\|=\|Q\|$ for some $\left(y_{0}^{*}, y_{0}\right) \in \Pi(F)$ and $y_{1} \in B_{F}$, where $v_{F}(Q)$ is the numerical radius of the map $\left.Q\right|_{F}: B_{F} \rightarrow F$.

Suppose that there is a sequence $\left\{\left(x_{k}, x_{k}^{*}\right)\right\}_{k=1}^{\infty}$ in $\Pi(X)$ such that $\lim _{k}\left|x_{k}^{*}\left(Q\left(x_{k}\right)\right)\right|=v(Q)$. Then

$$
\left|\left\langle x_{k}^{*}, Q\left(x_{k}\right)\right\rangle\right|=\left|\left\langle\left. x_{k}^{*}\right|_{F}, Q\left(\pi\left(x_{k}\right)\right)\right\rangle\right| \longrightarrow v(Q) \text {. }
$$

We may assume that the sequence $\left\{\left(\pi\left(x_{k}\right),\left.x_{k}^{*}\right|_{F}\right)\right\}_{k=1}^{\infty}$ converges to $\left(y, y^{*}\right) \in B_{F} \times B_{F^{*}}$ in the norm topology. So $v(Q)=\left|y^{*}(Q(y))\right| \geq v_{F}(Q)$. Since $\pi$ is parallel to $\Pi(X)$, $\left|\left\langle y^{*}, y\right\rangle\right|=\left\|y^{*}\right\| \cdot\|y\|$. By Lemma 5 ,

$$
v(Q)=\left|y^{*}(Q(y))\right| \leq v_{F}(Q) .
$$

So $v(Q)=\left|y^{*}(Q(y))\right|=v_{F}(Q)$. Since $\left.Q\right|_{F}$ is a numerical peak function, $\|y\|=1=\left\|y^{*}\right\|$ and $y=y_{0}$ and $y^{*}=y_{0}^{*}$.

Since $\pi$ is strong, $\lim _{n} x_{n}=y_{0}$. Let $x^{*}$ be the weak-* limit point of the sequence $\left\{x_{n}^{*}\right\}$. Then $x^{*}(y)=1$ and $\left\|x^{*}\right\|=1=$ $\left\|\left.x^{*}\right|_{F}\right\|$, and

$$
v(Q)=\left|x^{*}(Q(y))\right|=\left|y^{*}(Q(y))\right|=v_{F}(Q)
$$

implies that $\left.x^{*}\right|_{F}=y^{*}$ since $\left.Q\right|_{F}$ is a numerical strong peak function. Hence $x^{*}$ is unique because $X$ is smooth. Therefore $\left\{x_{n}^{*}\right\}_{n=1}^{\infty}$ converges weak-* to $x^{*}$. The proof is complete.

Theorem 7. Suppose that a Banach space $X$ space has the (FPA)-property with $\left\{\pi_{i}, F_{i}\right\}_{i \in I}$ and the corresponding projections are strong and parallel to $\Pi(X)$. One also assumes that each $\pi_{i}^{*}: X^{*} \rightarrow X^{*}$ is strong. Then the set of all very strong numerical and norm strong peak functions is dense in $A_{w u}\left(B_{X}: X\right)$.
Proof. By Proposition 3, the set of all polynomials $Q$ such that there exists norm-one projection $\pi:=\pi_{i}: X \rightarrow F$ such that $\pi \circ Q \circ \pi=Q$ and $\left.Q\right|_{F}$ is a norm and numerical peak function as a mapping from $B_{F}$ to $F$ is dense in $A_{w u}\left(B_{X}: X\right)$.

Fix corresponding $Q$ and $\pi$ and assume that $v_{F}(Q)=$ $\left|y_{0}^{*}\left(Q\left(y_{0}\right)\right)\right|$ and $\left\|Q\left(y_{1}\right)\right\|=\|Q\|$ for some $\left(y_{0}^{*}, y_{0}\right) \in \Pi(F)$ and $y_{1} \in B_{F}$, where $v_{F}(Q)$ is the numerical radius of the map $\left.Q\right|_{F}: B_{F} \rightarrow F$.

Suppose that there is a sequence $\left\{\left(x_{k}, x_{k}^{*}\right)\right\}_{k=1}^{\infty}$ in $\Pi(X)$ such that $\lim _{k}\left|x_{k}^{*}\left(Q\left(x_{k}\right)\right)\right|=v(Q)$. Then

$$
\left|\left\langle x_{k}^{*}, Q\left(x_{k}\right)\right\rangle\right|=\left|\left\langle\left. x_{k}^{*}\right|_{F}, Q\left(\pi\left(x_{k}\right)\right)\right\rangle\right| \longrightarrow v(Q) .
$$

We may assume that the sequence $\left\{\left(\pi\left(x_{k}\right),\left.x_{k}^{*}\right|_{F}\right)\right\}_{k=1}^{\infty}$ converges to $\left(y, y^{*}\right) \in B_{F} \times B_{F^{*}}$ in the norm topology. So $v(Q)=\left|y^{*}(Q(y))\right| \geq v_{F}(Q)$. Since $\pi$ is parallel to $\Pi(X)$, $\left|\left\langle y^{*}, y\right\rangle\right|=\left\|y^{*}\right\| \cdot\|y\|$. By Lemma 5,

$$
v(Q)=\left|y^{*}(Q(y))\right| \leq v_{F}(Q) .
$$

So $v(Q)=\left|y^{*}(Q(y))\right|=v_{F}(Q)$. Since $\left.Q\right|_{F}$ is a numerical peak function, $\|y\|=1=\left\|y^{*}\right\|$ and $y=y_{0}$ and $y^{*}=y_{0}^{*}$.

Since $\pi$ is strong, $\lim _{n} x_{n}=y_{0}$. Fix $z^{*} \in S_{X^{*}}$ to be a HahnBanach extension of $y^{*}$. Let $x^{*}$ be the weak-* limit point of the sequence $\left\{x_{n}^{*}\right\}_{n=1}^{\infty}$. Then $x^{*}(y)=1$ and $\left\|x^{*}\right\|=1=$ $\left\|\pi^{*}\left(x^{*}\right)\right\|$ and

$$
v(Q)=\left|x^{*}(Q(y))\right|=\left|y^{*}(Q(y))\right|=v_{F}(Q)
$$

implies that $\left.\pi^{*}\left(x^{*}\right)\right|_{F}=y^{*}$ since $\left.Q\right|_{F}$ is a numerical strong peak function so $\pi^{*}\left(x^{*}\right)=\pi^{*}\left(x^{*}\right)$.

Hence $\lim _{n} \pi^{*}\left(x_{n}^{*}\right)=\pi^{*}\left(z^{*}\right)$ and $\left\|\pi^{*}\left(z^{*}\right)\right\|=1$. Now we get $\left\|x_{n}^{*}-\pi^{*}\left(z^{*}\right)\right\| \rightarrow 0$ by the assumption. This shows that $\lim _{n}\left\|x_{n}^{*}-\pi^{*}\left(z^{*}\right)\right\|=0$. Therefore $x^{*}=\pi^{*}\left(z^{*}\right)$ and $Q$ is a very strong numerical peak function at $\left(y, \pi^{*}\left(z^{*}\right)\right)$. This completes the proof.

Corollary 8. Suppose that $X=\ell_{p}$ with $1<p<\infty$. Then the set of all very strong numerical and norm strong peak functions is dense in $A_{w u}\left(B_{X}: X\right)$.

Proof. Let $\left\{\pi_{i}, F_{i}\right\}_{i=1}^{\infty}$ be a projection consisting of $i$ th natural projections. Then these projections satisfy the conditions in Theorem 7. The proof is done.

\section{Generalized Numerical Index}

Proposition 9. Let $X$ be a (real or complex) Banach spaces and let $H$ be a closed subspace of $C_{b}\left(B_{X}: X\right)$. If $X$ has the (FPA)-property with $\left\{\pi_{i}, F_{i}\right\}_{i \in I}$, then $N(H) \geq \inf _{i \in I} N\left(H_{F_{i}}\right)$. In particular, $n^{(k)}(X) \geq \inf _{i \in I} n^{(k)}\left(F_{i}\right)$ for each $k \geq 1$.

Proof. Let $f \in S_{H}$. Given $\epsilon>0$, there is a norm one projection $\pi$ with a finite dimensional range $F$ such that $\|\pi \circ f \circ \pi\| \geq 1-\epsilon$. Let $g=\left.\pi \circ f \circ \pi\right|_{F}$ as a map in $H_{F}$ and

$$
v_{F}(g) \geq N\left(H_{F}\right)\|g\| \geq N\left(H_{F}\right)(1-\epsilon) .
$$


Then there is $\left(y, y^{*}\right) \in \Pi\left(H_{F}\right)$ such that $v_{H_{F}}(g)=\left|y^{*}(g(y))\right|$ since $F$ is finite dimensional. Notice that $\left(y, \pi^{*}\left(y^{*}\right)\right) \in \Pi(X)$ and so

$$
v_{F}(g)=\left|\pi^{*} y^{*}(f(\pi(y)))\right|=\left|\pi^{*} x^{*}(f(y))\right| \leq v_{H}(f) .
$$

Hence $v_{H}(f) \geq(1-\epsilon) N\left(H_{F}\right) \geq(1-\epsilon) \inf _{i \in I} N\left(H_{F_{i}}\right)$. Therefore $N(H) \geq \inf _{i \in I} N\left(H_{F_{i}}\right)$.

Proposition 10. Let $X$ be a complex Banach space and let $H$ be a subspace of $A_{b}\left(B_{X}: X\right)$ with a numerical boundary $\Gamma$. Suppose that a norm-one finite dimensional projection $(\pi, F)$ is parallel to $\Gamma$. Then for any $f \in H_{F}$,

$$
v_{F}(f)=v_{X}(f \circ \pi),
$$

where $v_{X}(f \circ \pi)$ is a numerical radius as a function $f \circ \pi$ : $B_{X} \rightarrow X$.

Proof. It is clear that $v_{F}(f) \leq v_{X}(f \circ \pi)$. For the converse, choose a sequence $\left\{\left(x_{n}, x_{n}^{*}\right)\right\}_{n=1}^{\infty}$ in $\Gamma$ such that

$$
v_{X}(f \circ \pi)=\lim _{n}\left|x_{n}^{*}\left(f\left(\pi\left(x_{n}\right)\right)\right)\right|=\lim _{n}\left\langle\left. x_{n}^{*}\right|_{F}, f\left(\pi\left(x_{n}\right)\right)\right\rangle .
$$

Since $\left\{\pi\left(x_{n}\right)\right\}_{n=1}^{\infty}$ is in the finite dimensional space $F$, we may assume that $\left\{\pi\left(x_{n}\right)\right\}_{n=1}^{\infty}$ converges to $y \in B_{F}$ and $\left\{\left.x_{n}^{*}\right|_{F}\right\}_{n=1}^{\infty}$ converges to $y^{*} \in B_{F^{*}}$. Then $\left|\left\langle y^{*}, y\right\rangle\right|=\left\|y^{*}\right\| \cdot\|y\|$. Thus by Lemma 5,

$$
v_{X}(f \circ \pi)=\left|y^{*}(f(y))\right| \leq v_{F}(f) .
$$

The proof is complete.

For the real Banach spaces, we get the following lemma for a homogeneous polynomial.

Lemma 11. Let $X$ be a real or complex Banach space, and let $f$ be a $k$-homogeneous polynomial. If there are $y \in B_{X}$ and $y^{*} \in$ $B_{X^{*}}$ such that $\left|y^{*}(y)\right|=\left\|y^{*}\right\| \cdot\|y\|$, then $\left|y^{*}(f(y))\right| \leq v(f)$.

Proof. If $y^{*}=0$, then it is clear. So we may assume that $y^{*} \neq 0$. We may assume that $y \neq 0$. The

$$
\begin{aligned}
\left|y^{*}(f(y))\right| & \leq\left|\frac{y^{*}}{\left\|y^{*}\right\|\|y\|^{k}}(f(y))\right| \\
& =\left|\frac{y^{*}}{\left\|y^{*}\right\|}\left(f\left(\frac{y}{\|y\|}\right)\right)\right| \leq v(f) .
\end{aligned}
$$

This completes the proof.

If we use Lemma 11 instead of Lemma 5 in the proof of Proposition 10, we get the following.

Proposition 12. Let $X$ be a real or complex Banach space, and let $\Gamma$ be a numerical boundary of $\mathscr{P}\left({ }^{k} X: X\right)$, where $k$ is a natural number. Suppose that a norm-one finite dimensional projection $(\pi, F)$ is parallel to $\Gamma$. Then for any $f \in \mathscr{P}\left({ }^{k} F: F\right)$,

$$
v_{F}(f)=v_{X}(f \circ \pi),
$$

where $v_{X}(f \circ \pi)$ is a numerical radius as a function $f \circ \pi$ : $B_{X} \rightarrow X$.

Now we get the extensions of the results of Ed-dari [13] and Kim [17] in the complex case.

Theorem 13. Let $X$ be a complex Banach space, and let $H$ be a subspace of $A_{b}\left(B_{X}: X\right)$ with a numerical boundary $\Gamma$. Suppose that the Banach space $X$ has the (FPA)-property with $\left\{\pi_{i}, F_{i}\right\}_{i \in I}$ and that the corresponding projections are parallel to $\Gamma$. Then

$$
N(H)=\inf _{i \in I} N\left(H_{F_{i}}\right) .
$$

In fact, $N(H)$ is a decreasing limit of the right-hand side with respect to the inclusion partial order.

Proof. For any $f \in H_{F}, v_{F_{i}}(f)=v_{X}\left(f \circ \pi_{i}\right)$ by Proposition 10 . $v_{F_{i}}(f)=v_{X}(f \circ \pi) \geq\|f \circ \pi\| N(H)=\|f\| N(H)$. Hence $N\left(H_{F_{i}}\right) \geq N(H)$ and it is easy to see that if $F_{i} \subset F_{j}$, then $N(H) \leq N\left(H_{F_{j}}\right) \leq N\left(H_{F_{i}}\right)$. Hence $N(H) \leq \inf _{i \in I} N\left(H_{F_{i}}\right)$. The converse is clear by Proposition 9.

For the general case we get a similar result about the polynomial numerical index if we use Proposition 12 in the proof of Theorem 13.

Theorem 14. Let $X$ be a real or complex Banach space, and let $\Gamma$ be a numerical boundary of $\mathscr{P}\left({ }^{k} X: X\right)$, where $k$ is a natural number. Suppose that $X$ has the (FPA)-property with $\left\{\pi_{i}, F_{i}\right\}_{i \in I}$ and that the corresponding projections are parallel to $\Gamma$. Then

$$
n^{(k)}(X)=\inf _{i \in I} n^{(k)}\left(F_{i}\right) .
$$

In fact, $n^{(k)}(X)$ is a decreasing limit of the right-hand side with respect to the inclusion partial order.

Proposition 15. Let $X$ be a real Banach space, and let $\Gamma$ be a numerical boundary of $\mathscr{P}\left({ }^{k} X: X\right)$, where $k$ is a natural number. Suppose that $X$ has the (FPA)-property with $\left\{\pi_{i}, F_{i}\right\}_{i \in I}$ and that the corresponding projections are parallel to $\Gamma$. If $n^{(k)}(X)=1$ and $k \geq 2$, then $X$ is one-dimensional.

Proof. We will use the fact [20] that if $X$ is a real finitedimensional Banach space with $n^{(k)}(X)=1$ and $k \geq 2$, then $X$ is one-dimensional. By Theorem 14 , we get $1=n^{(k)}(X)=$ $\inf _{i \in I} n^{(k)}\left(F_{i}\right)$ and $n^{(k)}\left(F_{i}\right)=1$ for all $i \in I$ and $F_{i}$ 's are onedimensional. Suppose on the contrary that $X$ is not onedimensional. Then we can choose two dimensional subspace $G$, and there is $i \in I$ with $\left\|i d_{G}-\left.\pi_{i}\right|_{G}\right\| \leq 1 / 2$. Then there are $x^{*} \in S_{X^{*}}$ and $a \in X$ such that $\pi_{i}(x)=x^{*}(x) a$ for all $x \in X$. Because $G$ is two-dimensional, there exists $w \in G \cap S_{X}$ with $x^{*}(w)=0$. So $\left\|w-\pi_{i}(w)\right\|=\|w\| \leq 1 / 2$, which is a contradiction to $\|w\|=1$. Therefore, $X$ is one-dimensional, and the proof is done.

Example 16. Let $\left(F_{i}\right)_{i=1}^{\infty}$ be a sequence of finite-dimensional Banach spaces, and consider the following spaces. For each $1<p<\infty$,

$$
X_{p}:=\left\{\left(x_{i}\right)_{i=1}^{\infty}: x_{i} \in F_{i},\left(\left\|x_{i}\right\|\right)_{i=1}^{\infty} \in \ell_{p}\right\}
$$


with the norm

$$
\left\|\left(x_{i}\right)_{i=1}^{\infty}\right\|=\left(\sum_{i=1}^{\infty}\left\|x_{i}\right\|^{p}\right)^{1 / p}
$$

is a Banach space with the shrinking and monotone finitedimensional decomposition with the projections

$$
P_{n}\left(\left(x_{i}\right)_{i=1}^{\infty}\right)=\left(x_{1}, \ldots, x_{n}, 0,0, \ldots\right), \quad(n \in \mathbb{N}) .
$$

The space $X_{0}:=\left\{\left(x_{i}\right)_{i=1}^{\infty}: x_{i} \in F_{i},\left(\left\|x_{i}\right\|\right)_{i=1}^{\infty} \in c_{0}\right\}$ with the norm $\left\|\left(x_{i}\right)_{i=1}^{\infty}\right\|=\sup _{i \in \mathbb{N}}\left\|x_{i}\right\|$ is also a Banach space with the shrinking and monotone finite-dimensional decomposition with the same projections $P_{n}$. Then it is easy to check that $\Pi\left(X_{p}\right)$ is parallel to the projections $\left(P_{n}, P_{n}\left(X_{p}\right)\right)$ for each $1<$ $p<\infty$ and $p=0$. So we get the following result. For each $k \in \mathbb{N}$ and each $1<p<\infty$ (or $p=0)$,

$$
n^{(k)}\left(X_{p}\right)=\lim _{n \rightarrow \infty} n^{(k)}\left(X_{p}^{n}\right)
$$

where $X_{p}^{n}=F_{1} \oplus_{p} F_{2} \oplus_{p} \cdots \oplus_{p} F_{n}$ and for complex Banach spaces; we get

$$
N\left(A\left(B_{X_{p}}: X_{p}\right)\right)=\lim _{n \rightarrow \infty} N\left(A\left(B_{X_{p}^{n}}: X_{p}^{n}\right)\right) .
$$

Corollary 17. Let $k \geq 1$ be a natural number and $1<p<\infty$. Then for a real or complex case,

$$
\lim _{m \rightarrow \infty} n^{(k)}\left(\ell_{p}^{m}\right)=n^{(k)}\left(\ell_{p}\right) \leq n^{(k)}\left(L_{p}(0,1)\right) .
$$

For the complex case we get

$$
\begin{aligned}
& \lim _{m \rightarrow \infty} N\left(A_{b}\left(B_{\ell_{p}^{m}}: \ell_{p}^{m}\right)\right) \\
& \quad=N\left(A_{b}\left(B_{\ell_{p}}: \ell_{p}\right)\right) \leq N\left(A_{b}\left(B_{L_{p}(0,1)}: L_{p}(0,1)\right)\right) .
\end{aligned}
$$

Proof. We give only the first part, since the proof of the next is similar. Let $H=\mathscr{P}\left({ }^{k} \ell_{p}\right)$. Then $\ell_{p}$ has the (FPA)-property with projections $\left\{\pi_{i}, \ell_{p}^{i}\right\}_{i=1}^{\infty}$, where each $\pi_{i}$ is the $i$ th natural projection. Notice that given projections are parallel to $\Pi(X)$. Hence $N(H)=\inf _{i \in I} N\left(H_{F_{i}}\right)$ by Theorem 13. Notice that $H_{F_{i}}$ is isometrically isomorphic to $\mathscr{P}\left({ }^{k} \ell_{p}^{i}\right)$.

On the other hand, if we let $H=\mathscr{P}\left({ }^{k} L_{p}(0,1)\right)$. Then $L_{p}(0,1)$ has (FPA)-property with projections $\left\{\pi_{i}, F_{i}\right\}$, where each $\pi_{i}$ is the conditional expectation with respect to the sub$\sigma$-algebra generated by finitely many disjoint subsets. Hence $N(H) \geq \inf _{i \in I} N\left(H_{F_{i}}\right)$. Notice also that $F_{i}$ is isometrically isomorphic to $\ell_{p}^{m}$ for some $m$. So $H_{F_{i}}$ is isometrically isomorphic to $\mathscr{P}\left({ }^{k} \ell_{p}^{m}\right)$. The proof is complete.

\section{Acknowledgments}

The first author was supported by the Basic Science Research Program through the National Research Foundation of Korea (NRF) funded by the Ministry of Education, Science and Technology (2010-0009854). The second author is the corresponding author and he was supported by the Basic Science Research Program through the National Research Foundation of Korea (NRF) funded by the Ministry of Education, Science and Technology (NRF-2012R1A1A1006869).

\section{References}

[1] S. Dineen, Complex Analysis on Infinite-Dimensional Spaces, Springer, London, UK, 1999.

[2] L. A. Harris, "The numerical range of holomorphic functions in Banach spaces," American Journal of Mathematics, vol. 93, pp. 1005-1019, 1971.

[3] M. D. Acosta and S. G. Kim, "Denseness of holomorphic functions attaining their numerical radii," Israel Journal of Mathematics, vol. 161, pp. 373-386, 2007.

[4] S. G. Kim, "Numerical peak points and numerical Šilov boundary for holomorphic functions," Proceedings of the American Mathematical Society, vol. 136, no. 12, pp. 4339-4347, 2008.

[5] S. G. Kim and H. J. Lee, "Numerical peak holomorphic functions on Banach spaces," Journal of Mathematical Analysis and Applications, vol. 364, no. 2, pp. 437-452, 2010.

[6] Y. S. Choi and S. G. Kim, "Norm or numerical radius attaining multilinear mappings and polynomials," Journal of the London Mathematical Society, vol. 54, no. 1, pp. 135-147, 1996.

[7] J. Bourgain, "On dentability and the Bishop-Phelps property," Israel Journal of Mathematics, vol. 28, no. 4, pp. 265-271, 1977.

[8] C. Stegall, "Optimization and differentiation in Banach spaces," Linear Algebra and Its Applications, vol. 84, pp. 191-211, 1986.

[9] J. Diestel and J. J. Uhl Jr., Vector Measures, American Mathematical Society, Providence, RI, USA, 1977.

[10] M. D. Acosta, J. Alaminos, D. García, and M. Maestre, "On holomorphic functions attaining their norms," Journal of Mathematical Analysis and Applications, vol. 297, no. 2, pp. 625-644, 2004.

[11] Y. S. Choi, H. J. Lee, and H. G. Song, "Bishop's theorem and differentiability of a subspace of $\mathrm{C}_{b}(\mathrm{~K})$," Israel Journal of Mathematics, vol. 180, no. 1, pp. 93-118, 2010.

[12] M. D. Acosta and S. G. Kim, "Numerical boundaries for some classical Banach spaces," Journal of Mathematical Analysis and Applications, vol. 350, no. 2, pp. 694-707, 2009.

[13] E. Ed-dari, "On the numerical index of Banach spaces," Linear Algebra and Its Applications, vol. 403, pp. 86-96, 2005.

[14] Y. S. Choi, D. Garcia, S. G. Kim, and M. Maestre, "The polynomial numerical index of a Banach space," Proceedings of the Edinburgh Mathematical Society, vol. 49, no. 1, pp. 39-52, 2006.

[15] D. García, B. C. Grecu, M. Maestre, M. Martín, and J. Merí, "Two-dimensional Banach spaces with polynomial numerical index zero," Linear Algebra and Its Applications, vol. 430, no. 89, pp. 2488-2500, 2009.

[16] S. G. Kim, "Norm and numerical radius of 2-homogeneous polynomials on the real space $l_{p}^{2}, 1<p<\infty$," Kyungpook Mathematical Journal, vol. 48, no. 3, pp. 387-393, 2008.

[17] S. G. Kim, “The polynomial numerical index of $L_{p}(\mu)$," Kyungpook Mathematical Journal, vol. 53, no. 1, pp. 117-124, 2013.

[18] J. Kim and H. J. Lee, "Strong peak points and strongly norm attaining points with applications to denseness and polynomial numerical indices," Journal of Functional Analysis, vol. 257, no. 4, pp. 931-947, 2009.

[19] S. G. Kim, M. Martín, and J. Merí, "On the polynomial numerical index of the real spaces $c_{0}$ and $l_{1}, l_{\infty}$," Journal of Mathematical Analysis and Applications, vol. 337, no. 1, pp. 98106, 2008.

[20] H. J. Lee, "Banach spaces with polynomial numerical index 1," Bulletin of the London Mathematical Society, vol. 40, no. 2, pp. 193-198, 2008. 
[21] J. Lindenstrauss and L. Tzafriri, Classical Banach Spaces. II, Springer, 1979.

[22] J. Johnson and J. Wolfe, "Norm attaining operators," Polska Akademia Nauk. Institut Matematyczny. Studia Mathematica, vol. 65, no. 1, pp. 7-19, 1979. 


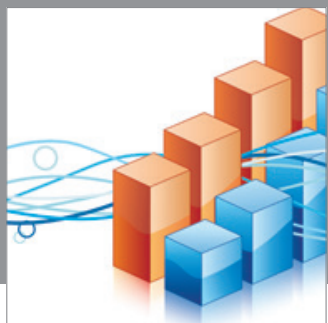

Advances in

Operations Research

mansans

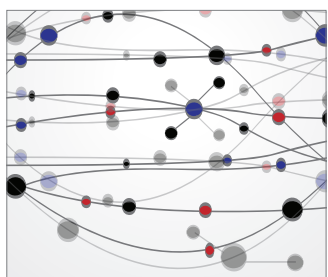

The Scientific World Journal
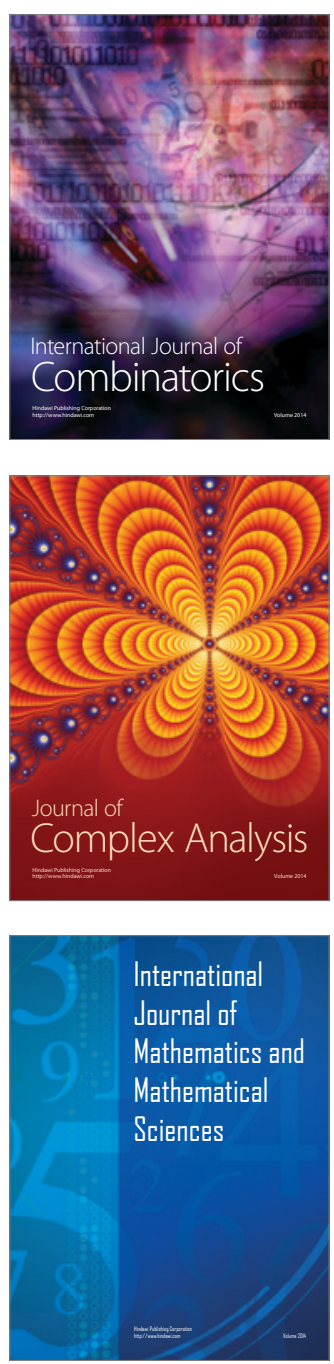
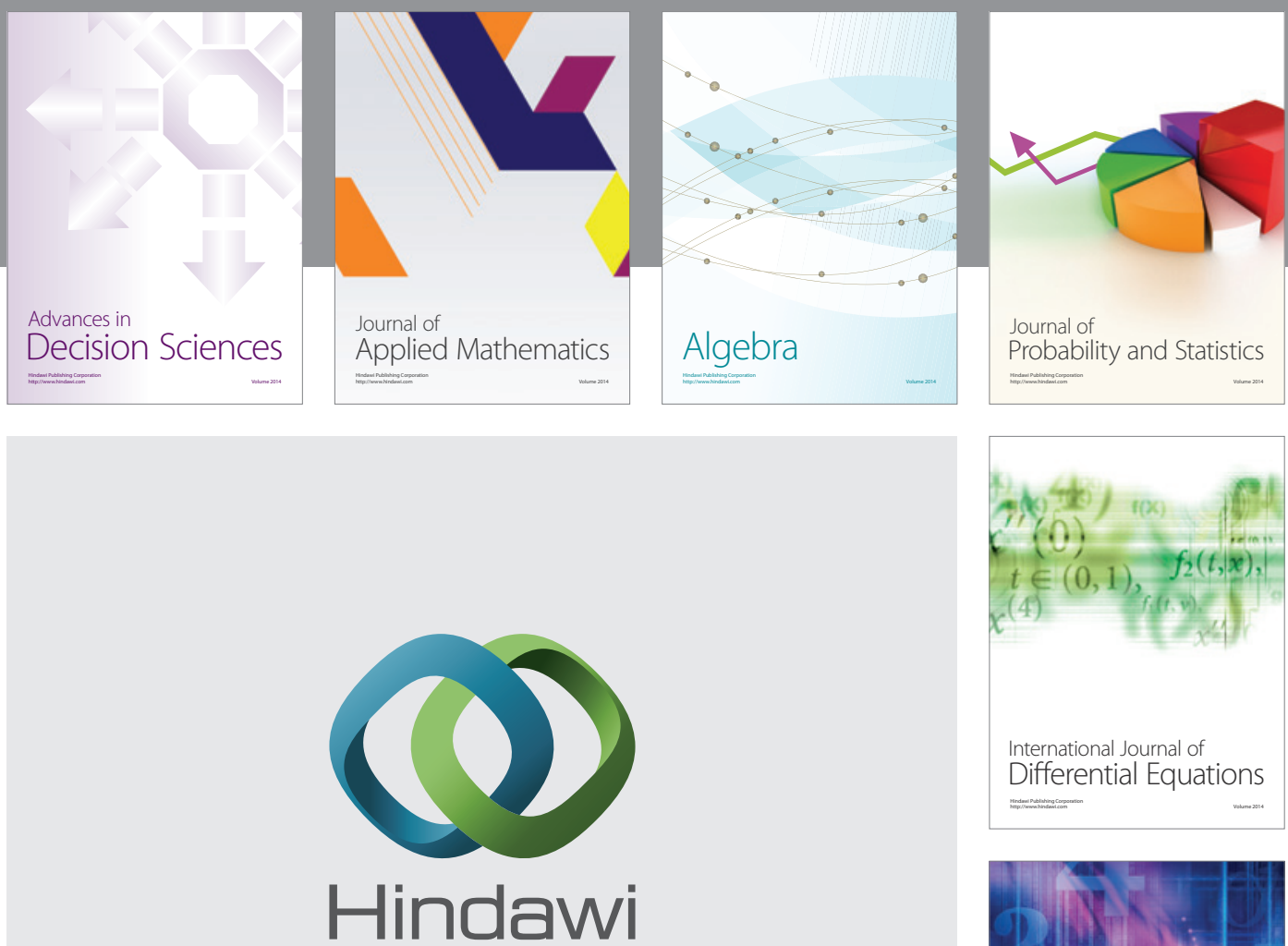

Submit your manuscripts at http://www.hindawi.com
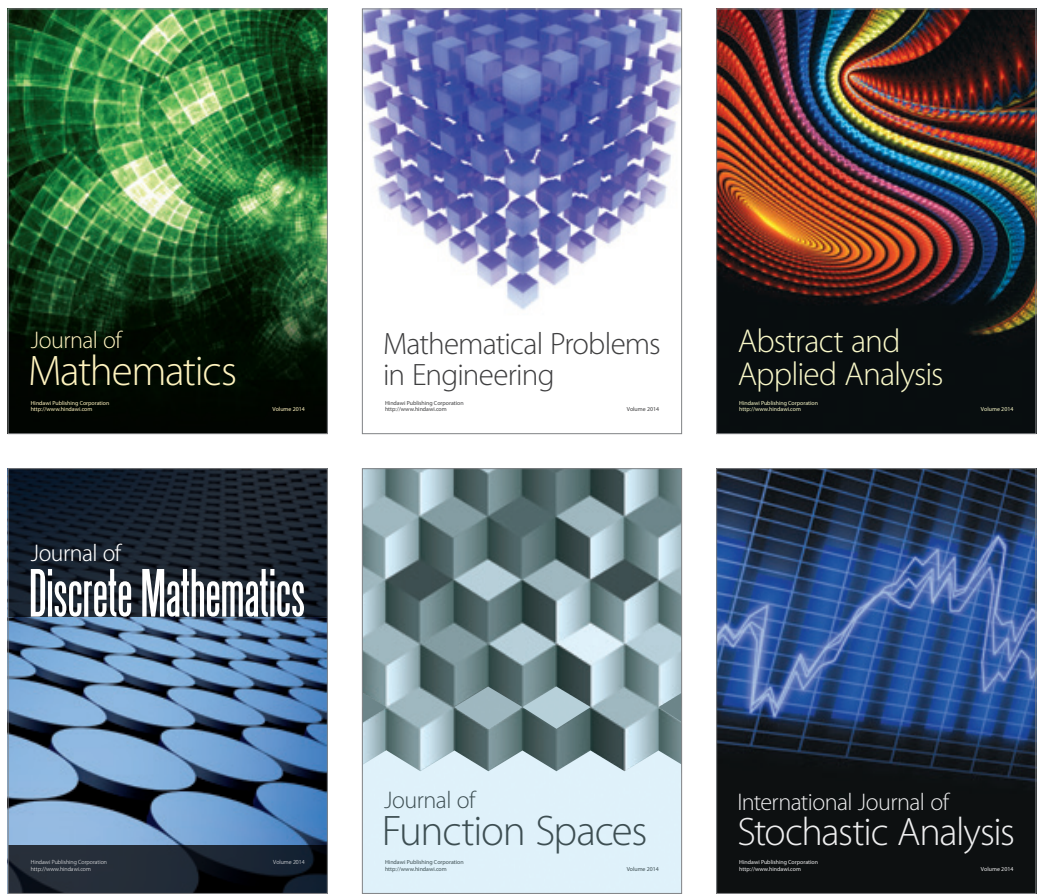

Journal of

Function Spaces

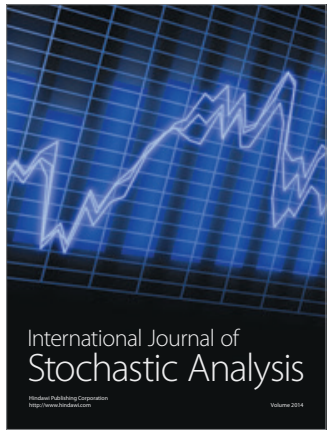

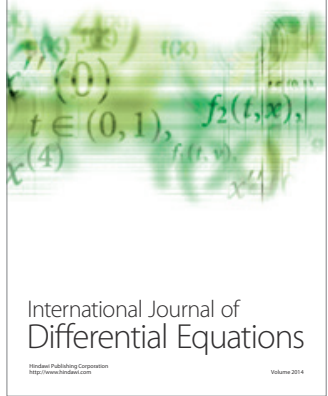
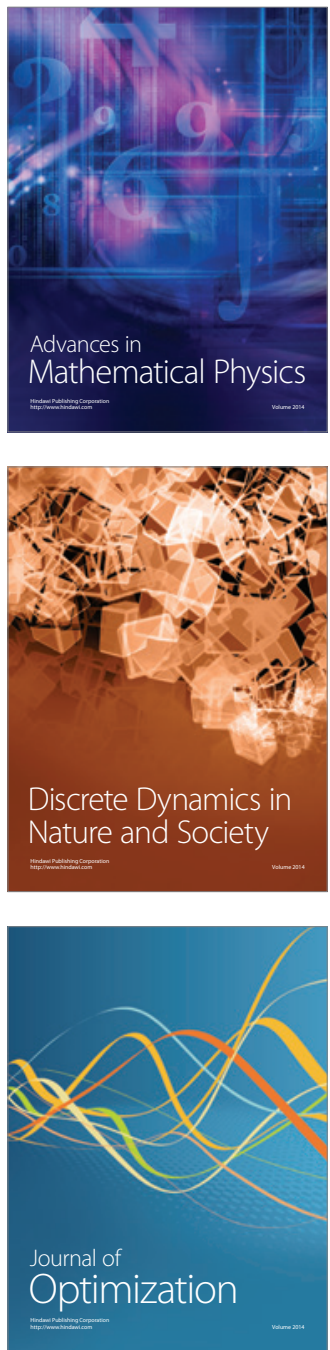